\title{
Norois
}

Environnement, aménagement, société

198 | 2006/1

Géosymbole, écologie, renouvellement urbain, modélisation

\section{Origine et intérêt écologique des sols limoneux des dépressions humides du massif dunaire d'Hatainville (Manche)}

Origin and ecological significance of silty soils of dune slacks of Hatainville dune massif (Manche)

Patrick Le Gouée, Yves Petit-Berghem et Audrey Folley

\section{(2) OpenEdition}

Journals

Édition électronique

URL : http://journals.openedition.org/norois/2053

DOI : $10.4000 /$ norois. 2053

ISBN : 978-2-7535-1546-8

ISSN : $1760-8546$

Éditeur

Presses universitaires de Rennes

Édition imprimée

Date de publication : 1 mars 2006

Pagination : 63-80

ISBN : 978-2-7535-0307-6

ISSN : 0029-182X

Référence électronique

Patrick Le Gouée, Yves Petit-Berghem et Audrey Folley, « Origine et intérêt écologique des sols

limoneux des dépressions humides du massif dunaire d'Hatainville (Manche) », Norois [En ligne], 198 | 2006/1, mis en ligne le 13 décembre 2008, consulté le 19 avril 2019. URL : http:// journals.openedition.org/norois/2053 ; DOI : 10.4000/norois.2053 


\title{
ORIGINE ET INTÉRÊT ÉCOLOGIQUE DES SOLS LIMONEUX DES DÉPRESSIONS HuMIDES DU MASSIF DUNAIRE D'HATAINVILLE (MANCHE)
}

\author{
Patrick Le Gouée, Yves Petit-Berghem, Audrey Folley \\ GÉOPHEN - Université de CAen Basse-Normandie \\ LETG, UMR 6554 - CNRS \\ patrick.legouee@unicaen.fr,yves.petit-berghem@unicaen.fr,folleyaudrey@wanadoo.fr
}

\begin{abstract}
RÉSUMÉ
Sur les côtes européennes, les massifs dunaires sont caractérisés par de nombreux habitats à forte valeur écologique qu'explique la présence d'une nappe phréatique. Depuis une vingtaine d'années, les plantes pionnières et prairiales de ces habitats subissent une tendance très importante à la régression. Le massif dunaire d'Hatainville en est un parfait exemple. La protection des espèces herbacées rares passe par une meilleure connaissance des sols et de leur fonctionnement. Une étude pédologique a été menée sur les sols limoneux des dépressions humides du massif, sols dont la découverte fut inattendue. Ces sols présentent en effet la particularité de détenir les espèces pionnières et prairiales les plus riches. Les résultats analytiques ont permis de préciser l'origine et l'intérêt écologique des sols limoneux. Ils soulignent également la nécessité de préserver ce matériel édaphique dans le cadre d'une gestion conservatoire de la biodiversité du massif dunaire.
\end{abstract}

MotS CLÉS : Biodiversité végétale - Cotentin occidental - Dépression dunaire humide - Gestion conservatoire - Sol limoneux.

\footnotetext{
ABSTRACT

Origin and ecological significance of silty soils of dune slacks of Hatainville dune massif (Manche)

Along the European coasts, dune massifs are characterized by numerous settlements with great ecological value in relation to phreatic water. Since about twenty years, pioneer and grassy species of those settlements are subjected to substantial decline. Dune massif of Hatainville is a perfect sample of this regressive trend. Protection of scarce grassy species requires a better knowledge of soils and their dynamics. Pedological investigation has been carried out concerning unexpected silty soils of massif dune slacks. These soils are original insofar as they own richest pioneer and grassy species. Origin and ecological value of the silty soils have been cleared by analytic data. These data also underline the need for preserving this edaphic material within the conservatory management of the biodiversity of dune massif.
}

KEY WORDS : Conservation Management - Dune Slack - Plant Biodiversity - Silty Soil - Western Cotentin. 
Sur les côtes européennes, les massifs dunaires sont caractérisés par la présence de nombreux habitats à forte valeur écologique en raison de l'existence de gradients hydriques et chimiques qui favorisent la diversité floristique et faunistique (Grootjans et al., 1998). Cependant, cette biodiversité est menacée de disparition par les activités touristiques, les pratiques agricoles et l'embroussaillement. Les remblaiements, les aménagements touristiques ou portuaires, l'urbanisation littorale, les modifications des conditions hydrologiques par drainage ou assèchement des marais arrière-dunaires et l'eutrophisation de l'eau liée au développement de l'agriculture intensive (cultures légumières) sont autant de facteurs agissant sur l'évolution régressive des habitats dunaires (Foucault, 1984). Cette tendance se retrouve également dans d'autres pays européens comme l'Espagne (Llamas, 1990) ou les Pays-Bas (Van Dijk et Grootjans, 1993) avec toujours les mêmes causes. Plus encore, ce sont les modifications hydrologiques observées aux Pays-Bas, en Belgique et France qui déstabilisent l'équilibre fragile des habitats les plus riches comme les mares dunaires, les pelouses pionnières ou bien encore les prairies humides dunaires (Natura 2000, 1999; Lammerts et al., 2001). L'abaissement constaté du niveau des nappes phréatiques assèche ces milieux qui subissent une tendance très importante à la régression depuis une vingtaine d'années (Natura 2000, 1999).

Le massif dunaire d'Hatainville, situé dans la partie occidentale du Cotentin, est également affecté par cette tendance. À la demande des organismes qui ont en charge la gestion de ce site, le Conservatoire du Littoral et le Syndicat Mixte des Espaces Littoraux de la Manche, une étude pédologique a été menée dans les dépressions dunaires humides afin de préciser les liens entre les sols et les espèces pionnières et prairiales les plus intéressantes. L'objectif initial portait sur l'acquisition de nouvelles connaissances afin d'améliorer les modalités de conservation des plantes herbacées protégées au niveau régional et national. La réalisation de micro-fosses et de mesures analytiques de laboratoire sur des échantillons prélevés ont permis de caractériser les propriétés édaphiques et de préciser la nature des constituants et organisations des horizons de tous les types de sol.

La découverte de sols limoneux portant exclusivement les espèces les plus précieuses, comme l'Ache rampante, Apium repens, ou la Littorelle des lacs, Littorella uniflora, a justifié une étude spécifique à ce matériel édaphique afin de cerner son origine et son intérêt écologique. Pour expliquer l'origine des limons, quelques hypothèses peuvent être avancées. L'installation de mares peu profondes dans les dépressions dunaires favoriserait l'installation d'écosystèmes aquatiques dans lesquels les Diatomées et les Characées trouvent leur place (Géhu et Géhu-Franck, 1982). Ces algues microscopiques se déposeraient à la surface des dépressions lors du retrait complet de la nappe : les limons seraient alors d'origine biogène. Cependant, comment justifier le fait que toutes les dépressions humides ne contiennent pas de limons? Comment expliquer aussi qu'à l'intérieur même d'une dépression, certaines zones soient limoneuses et d'autres pas? Une autre hypothèse est proposée. Historiquement, la valorisation du massif s'est orientée vers le pâturage extensif. On peut alors s'interroger sur l'origine anthropique des limons qui auraient été déposés dans des dépressions accessibles pour permettre la création d'îlots de pâtures à haute valeur fourragère. Toutefois, les archives communales n'évoquent rien à ce sujet.

L'article se propose donc d'expliquer la présence de ces sols originaux en contexte de dunes littorales, de comprendre en quoi ils peuvent être conservateurs de la richesse floristique du massif d'Hatainville et de démontrer la nécessité d'adapter les modalités de gestion actuelles concernant les sols limoneux.

\section{Le massif dunaire d'Hatainville}

\section{LOCALISATION ET CARACTÉRISTIQUES GÉOMORPHOLOGIQUES}

Le littoral du département de la Manche, l'un des plus étendus de France, possède plus de 350 kilomètres de rivages diversement occupés et mis en valeur par les sociétés. Dunes, falaises 
et havres alternent et confèrent au littoral sa diversité paysagère. Le Cotentin occidental offre plus de 100 kilomètres de dunes inégalement développées à l'intérieur des terres. Le site dunaire d'Hatainville est situé à une trentaine de kilomètres au Sud-Ouest de Cherbourg et fait partie d'un ensemble dunaire plus étendu situé entre les caps de Rozel et de Carteret (fig. la). Cet ensemble correspond à un champ de dune qui s'étend sur dix kilomètres de linéaire de côte et sur une superficie de 800 hectares environ. Les dunes d'Hatainville sont le résultat de l'histoire géomorphologique et climatique armoricaine et notamment de la remontée progressive du niveau marin liée à la transgression post-glaciaire. Les sables provenant de l'érosion des côtes et charriés par la mer ont été poussés par les vents d'ouest pour former un vaste massif plaqué sur l'ancienne falaise littorale en grande partie fossilisée. Atteignant $80 \mathrm{~m}$ d'altitude au maximum, les dunes d'Hatainville montrent une diversité de formes d'ampleur variable et constituent l'un des derniers grands ensembles de dunes perchées encore intact en Europe.

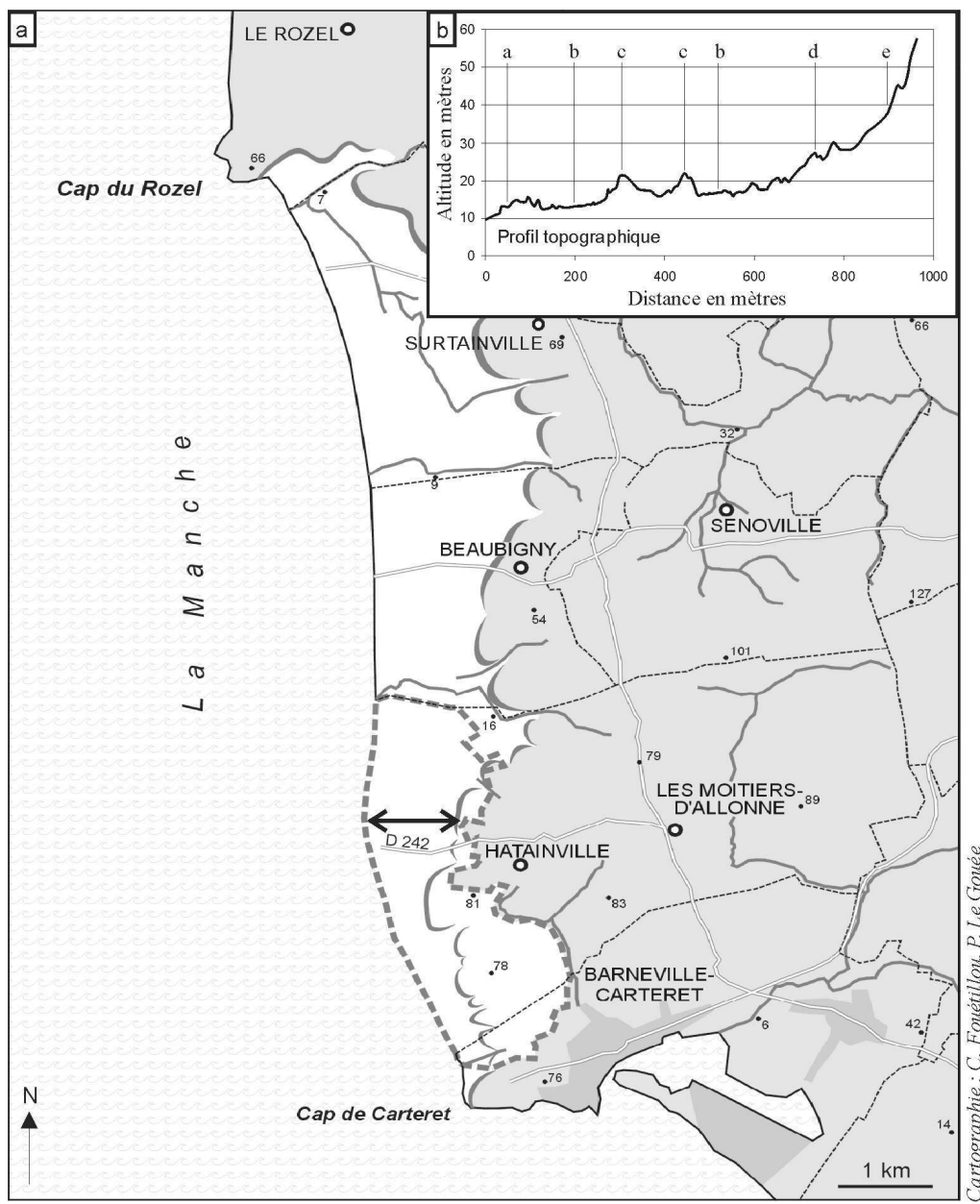

Figure 1 : Situation du massif dunaire d'Hatainville

Location of massif

a. Vue générale, b. Profil topographique (a : dunes embryonnaires et dunes vives, $\mathrm{b}$ : dunes basses, $\mathrm{c}$ : dunes paraboliques et longitudinales, $\mathrm{d}$ : talus, e : paléofalaise).

a. Global view, $b$ : Topographic profile (a : embryonal and active dunes, $b$ : low dunes, $c$ : longitudinal and parabolic dunes, $d$ : slope, $e$ : paleocliff).
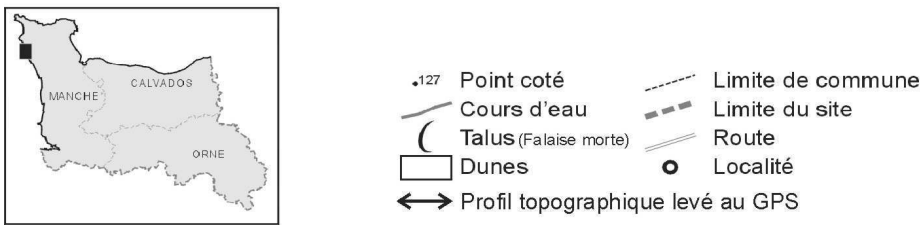
D'un point de vue topo-géomorphologique, le site peut être divisé en deux grands secteurs bien distincts : un secteur sud allant de la limite communale de Barneville-Carteret à la D 242 ; un secteur nord allant de la D 242 à la limite communale de Beaubigny. La partie méridionale comprend une aire sableuse pouvant atteindre $1500 \mathrm{~m}$ de largeur; les dunes basses sont rares tandis que la falaise morte atteste la présence de dunes plaquées et des valleuses qui sont à l'origine d'écoulements temporaires. Les dunes perchées s'élèvent entre 50 et $80 \mathrm{~m}$ d'altitude et laissent peu de place à la roche qui affleure cependant à certains endroits (grès et schistes du Permien). Les dépressions humides sont peu importantes et souvent transformées en mares expérimentales. Comme le montre le profil topographique levé au GPS monofréquence (fig. 1b), le secteur nord souligne la présence de dunes actives (dunes embryonnaires et vives bordant le rivage) parfois taillées en micro-falaises (Levoy, 1996) et de dunes paraboliques et longitudinales situées plus à l'arrière et en grande partie stabilisées par la végétation. Ce secteur est caractérisé également par de nombreuses dunes basses propices à la formation et au développement des dépressions humides (dépressions interdunaires). Mais ce sont surtout les dunes paraboliques, au profil conforme aux vents d'ouest dominants, qui vont générer à la base de leur front la plupart de ces dépressions (dépressions intradunaires).

\section{DES DÉPRESSIONS HUMIDES DE PETITE TAILLE}

L'une des caractéristiques majeures du massif d'Hatainville est la multitude de dépressions où l'eau s'accumule, temporairement le plus souvent (fig. 2a). En s'agrandissant, celles-ci donnent naissance à des dépressions de forme allongée et de faible profondeur. Contrairement à d'autres massifs dunaires (Vauville par exemple, située plus au nord), les dépressions rencontrées ne forment pas de grandes étendues aquatiques mais plutôt des petites surfaces isolées (quelques dizaines de $\mathrm{m}^{2}$ ) issues du remaniement des dunes anciennes. Certaines dépressions ont été recreusées puis transformées en mares ces dernières années avec des engins mécaniques. Ces mares de dimensions modestes $\left(50\right.$ à $100 \mathrm{~m}^{2}$ ) et de forme circulaire (Folley, 2004) servent d'abreuvoirs pour les animaux. Le fonctionnement hydrique des dépressions est étroitement lié à la présence d'un réservoir aquifère d'une petite nappe libre d'eau douce. Les eaux salines sont intrusives sous la lame d'eau douce avec une interface en biseau. La hauteur de la surface libre de la nappe d'eau douce oscille substantiellement avec les saisons. Elle dépend en grande partie des surplus hydrologiques ${ }^{1}$. Lorsque ceux-ci sont excédentaires (2000-2001) sur une période s'étalant d'octobre à mars, l'analyse des photographies obliques de la DIREN Basse-Normandie révèle que la surface libre de la nappe d'eau se situe au maximum $30 \mathrm{~cm}$ au-dessus du fond des dépressions. En année fortement déficitaire en pluies (2004-2005, faibles surplus hydrologiques entre décembre et février), le niveau d'eau ${ }^{2}$ se positionne alors $80 \mathrm{~cm}$ à $1,30 \mathrm{~m}$ sous les dépressions. La hauteur de la nappe d'eau et la durée de submersion des dépressions dunaires attestent donc d'une importante variabilité interannuelle.

\section{UNE FORTE BIODIVERSITÉ}

La flore du massif d'Hatainville est riche : 462 plantes parmi les 1307 êtres vivants sont citées dans l'étude de A. Livory et P. Stallegger (2001). La végétation des dépressions s'organise en ceintures plus ou moins concentriques (Provost, 1975). Dans les secteurs les plus fréquemment inondés, les plantes vivaces amphibies comme la Sagine noueuse, Sagina nodosa, ou la Littorelle des Lacs, Littorella uniflora, sont accompagnées par un grand nombre de mousses. D'autres

1. Volumes d'eau alimentant par drainage ou ruissellement la nappe phréatique locale. Ils sont calculés selon la méthode du bilan hydrique à partir des données locales. Surplus hydrologiques (S) en année moyenne (1990-2003) : 147 mm pour une réserve utile de $50 \mathrm{~mm}$. En année déficitaire (1995-1996), S = 79 mm et en année excédentaire (2000-2001), $\mathrm{S}=486 \mathrm{~mm}$.

2. Relevés du réseau piézométrique du SyMEL (Syndicat Mixte des Espaces Littoraux de la Manche). 
espèces rencontrées à ce niveau sont de très grande valeur patrimoniale (Gentiane amère, Gentianella amarella, et Pyrole à feuilles rondes, Pyrola rotundifolia, protégées au niveau national; Orchis à fleur lâche, Orchis laxiflora, Laîche à trois nervures, Carex trinervis, ou Germandrée des marais, Teucrium scordium, protégées au niveau régional; Provost, 1998). Lorsque les variations des niveaux d'eau sont plus importantes apparaissent des bas marais dunaires occupés par des jonchaies et des cariçaies renfermant des espèces prairiales hygrophiles comme l'Iris jaune, Iris pseudacorus, le Choin noirâtre, Schoenus nigricans, la Laîche noire, Carex nigra, ou le Jonc maritime, Juncus maritimus. Sur le pourtour des dépressions, la dynamique d'atterrissement peut conduire à la formation de roselières formées de plantes hautes et recouvrantes comme le Scirpe maritime, Scirpus maritimus, le Marisque (une seule station dans le massif), Mariscus, ou le Roseau à plumet, Phragmites communis. Si ces communautés d'espèces non spécifiquement dunaires sont intéressantes d'un point de vue paysager (Ducerf et Thiry, 2003), elles contribuent à fermer les dépressions qui tendent à s'enrichir en espèces ligneuses envahissantes (fourrés formés surtout par le Saule rampant, Salix repens, accompagné par le Troène, Ligustrum vulgare, le Prunellier, Prunus spinosa, ou l'Aubépine, Crataegus oxyacantha). Ces arbustes au tempérament déjà forestier (Favennec, 1998) proviennent des landes voisines ou des haies du bocage. Si la pression du bétail est faible, ces espèces banalisent le paysage et entraînent ainsi une perte de biodiversité. Inversement, une trop forte concentration d'animaux induit des apports en nitrates élevés entraînant l'arrivée de plantes nitrophiles (ronces, Rubus, sureaux, Sambucus nigra) préjudiciables à la qualité de la flore littorale.

\section{Les sols des dépressions humides dunaires}

\section{MATÉRIELS ET MÉTHOdES}

La caractérisation des sols des dépressions humides s'est appuyée sur une première phase prospective au cours de laquelle nous nous sommes attachés à sonder toutes les dépressions du massif d'Hatainville au moyen d'une tarière à main puis à décrire en détail le profil pédologique des microfosses représentatives des principaux types de sol. Dans une seconde phase analytique, les horizons des profils référents ont été échantillonnés afin de déterminer leurs propriétés. L'identification des constituants et organisations des horizons a été réalisée au moyen de lames minces de sol non remanié confectionnées selon la méthode de P. Guilloré (1980). Ces lames mammouths (14 x 6 cm) ont été étudiées au microscope polarisant optique (Olympus BX51) et ont fait l'objet de nombreux clichés photographiques. Au moyen du granulomètre laser (Coulter LS200), nous avons évalué la distribution statistique des particules minérales selon leur taille, ce qui a permis ensuite de préciser la texture de chaque horizon. Pour les horizons les plus sableux, la granulométrie a été effectuée par voie sèche selon la méthode des tamis. L'étude de la mouillabilité des humus (Dekker et Jungerius, 1990) et des humidités caractéristiques au moyen de la presse à membrane (Van Oort, 1984) a précisé le comportement hydrodynamique du matériel édaphique. Dans une dizaine de dépressions, des profils hydriques ont été dressés entre la nappe d'eau libre et l'épisolum afin de juger des dynamiques capillaires sous régime permanent (Hillel, 1974) susceptibles de palier le déficit en eau de la réserve utile. Tous les 15 jours, de mars à juin 2005, des carottages ont ainsi été réalisés dans 10 dépressions humides aux niveaux $-5,-10,-20,-40$ et $-60 \mathrm{~cm}$. Au cours des campagnes de prélèvement, la surface libre de la nappe s'est toujours située entre -80 et $-130 \mathrm{~cm}$. La réserve utile des horizons a été calculée en prenant en compte les teneurs en eau à la capacité au champ et au point de flétrissement permanent, les densités apparentes et les épaisseurs. Les propriétés chimiques $\left(\mathrm{pH}_{2} \mathrm{O}\right.$, Calcaire total et actif, $\mathrm{C}$ org., $\mathrm{C}$ inor., N, C/N, Fer total et Fer libre, CEC et V) résultent d'analyses menées au laboratoire Géophen et en laboratoire privé. L'effort de caractérisation chimique s'est porté plus particulièrement sur les sols considérés comme les plus originaux. Les observations de terrain et les bases de données analytiques ont permis de définir la pédogenèse et de qualifier les sols selon le Référentiel pédologique 1995 (Baize et Girard, 1995). 


\section{RÉSULTATS}

Trois types de sols ont été recensés dans les dépressions humides. Les données descriptives et analytiques de ces sols sont présentées dans les figures 2, 3 et 4. Le premier type, le plus couramment rencontré, s'apparente à des sols superficiels (fig. 2b) constitués d'un humus de 2 à $3 \mathrm{~cm}$ d'épaisseur de type moder carbonaté (Jabiol et al., 1995) et d'un horizon A carbonaté (fig. 4c) de moins de 10 cm d'épaisseur, à structure particulaire grossière (fig. 3a).

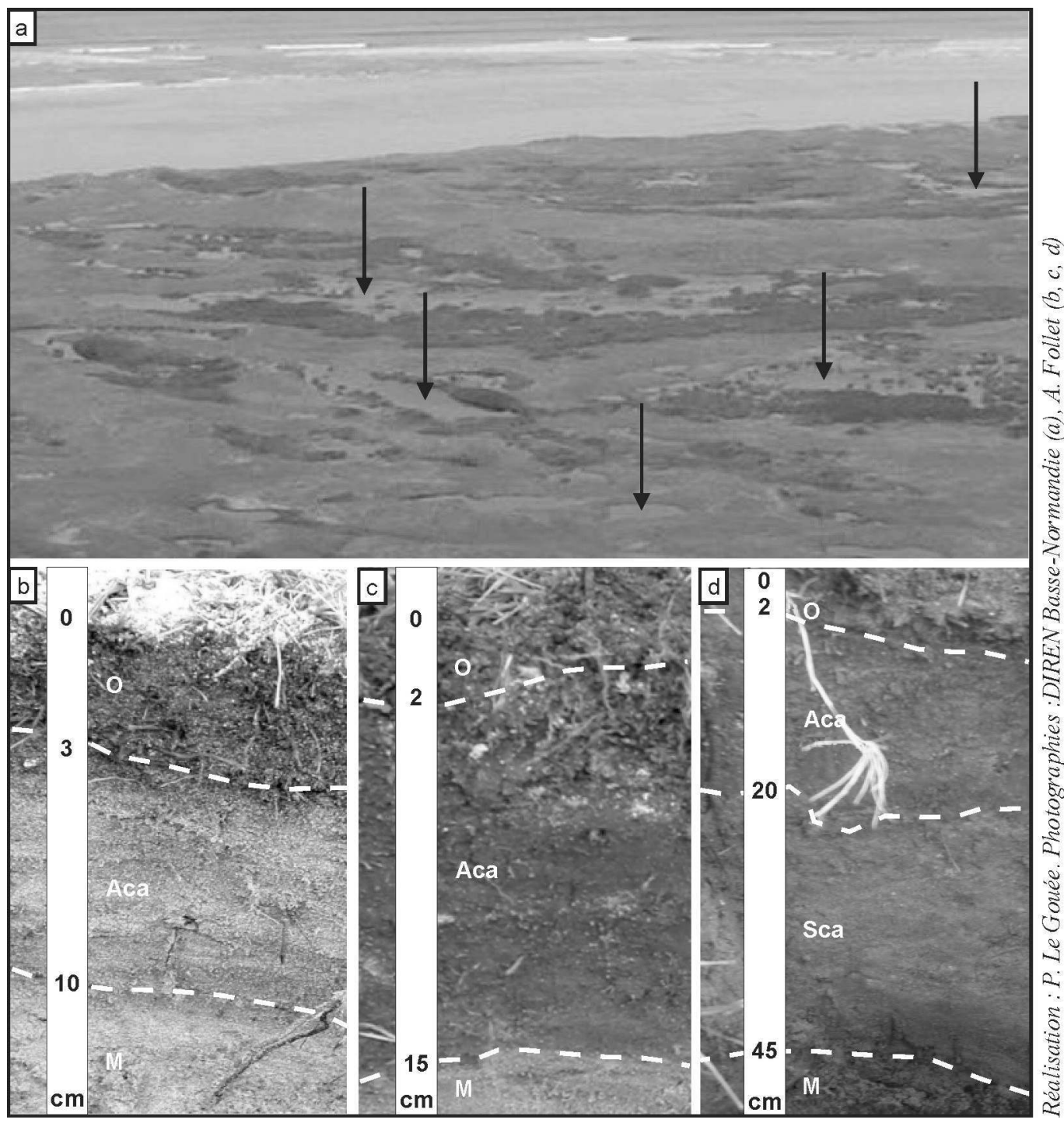

Figure 2 : Dépressions dunaires submergées (janvier 2001). Sols des dépressions dunaires

Submerged dune slacks (january 2001). Dune slacks soils

a. Dépressions dunaires submergées, b. Régosol sableux, c. Rendosol limoneux, d.Calcosol limoneux. O : horizon organique de sol minéral, Aca : horizon organo-minéral calcaire, Sca : horizon pédologique calcaire d'altération , $\mathrm{M}$ : sable dunaire meuble.

a. Submerged dune slacks, b. Sandy Regosol, c. Silty Rendosol, d. Silty Calcosol. O : organic horizon, Aca : calcareous organo-mineral horizon, Sca : calcareous pedological horizon of alteration, M : loose dune sand. 
Ces sols peu épais, reposant sur le sable dunaire, sont qualifiés de régosols. La granulométrie de l'horizon A souligne l'omniprésence de la fraction sableuse (fig. 3d). Les particules minérales des sols sont constituées de sables à plus de $99 \%$. Il s'agit surtout de sables fins et secondairement de sables moyens, représentant respectivement 67 et $30 \%$ des particules élémentaires de taille inférieure à $2 \mathrm{~mm}$. En raison d'une texture sableuse et d'une faible épaisseur, les régosols témoignent de réserves utiles très basses, légèrement supérieures à $10 \mathrm{~mm}$ (fig. 4c). La courbe des humidités (fig.4a) atteste d'une vidange brutale de la réserve utile dès la mise en place de conditions desséchantes. Ainsi, à pF 2,7, le taux de remplissage de la réserve utile n'est plus que de $10 \%$. La teneur en eau des régosols se situe alors au voisinage des valeurs hydriques contraignantes pour les végétaux (valeurs relatives au point de flétrissement temporaire à $\mathrm{pF} 4$ et permament à $\mathrm{pF}$ 4,2). L'alcalinité de ces sols est toujours vérifiée $(\mathrm{pH} \mathrm{8,4).} \mathrm{Le} \mathrm{taux} \mathrm{de} \mathrm{calcaire} \mathrm{total} \mathrm{de} \mathrm{l'horizon} \mathrm{A}$ (13\%) rend compte de régosols hypo-calcaires (moins de $15 \%$ de calcaire).

Les types 2 et 3 présentent les mêmes caractéristiques édaphiques mais se distinguent dans la nomenclature des couvertures pédologiques en raison d'une différence d'épaisseur. Il s'agit de sols peu à moyennement épais (fig. 2c et fig. 2d), de 20 à $50 \mathrm{~cm}$, constitués d'un humus de type dysmull carbonaté (fig. 4c) surmontant un horizon A carbonaté organo-minéral, de structure granulaire (fig. 3b). Lorsque l'épaisseur est supérieure à $40 \mathrm{~cm}$, il se développe un horizon $\mathrm{S}$ carbonaté à structure particulaire fine (fig. 3c). Ces sols, à texture sablo-limoneuse (fig. 3d), sont qualifiés de rendosol et calcosol (présence d'un horizon S). L'observation des profils de sol souligne la rupture structurale et texturale entre les horizons pédologiques et le sable dunaire sur lequel ils reposent (fig. 2c et fig. 2d). Les limons peuvent représenter ainsi 30 à $40 \%$ de la granulométrie totale des rendosols et calcosols. Dans le dernier cas, la fraction limoneuse des horizons A et $\mathrm{S}$ est analogue (fig. 3d).

La présence des limons ne contribue pas à augmenter la capacité de stockage en eau utile. En calculant la réserve utile par cm d'épaisseur de sol à partir des données de la figure 4c, on note que cette capacité est plus importante pour le régosol sableux $(1,91 \mathrm{~mm}$ de RU par cm de sol contre 1,86 pour le calcosol). La valeur du calcosol résulte des RU de l'horizon A et $\mathrm{S}$ qui sont respectivement de 2,1 et 1,7. Pour le calcosol comme pour tous les autres sols des dépressions dunaires, c'est la teneur en carbone organique qui agit principalement sur les valeurs de RU par $\mathrm{cm}$ de sol. L'impact de la teneur en carbone organique s'atténue progressivement lorsque l'épaisseur devient un critère majeur dans la distinction des couvertures pédologiques. C'est ainsi que le calcosol présente une RU de $80 \mathrm{~mm}$ contre $13 \mathrm{~mm}$ pour le régosol (fig. 4c). Au regard des réserves utiles, les conditions de stress hydrique semblent donc moins marquées pour les sols «épais ». Cependant, le facteur granulométrique ne doit pas être ignoré dans l'établissement du stress en eau car il intervient dans la dynamique de déstockage de la réserve utile en phase de dessèchement. D'après la figure $4 \mathrm{a}$, nous observons que le calcosol, à texture plus fine, rend compte d'une dynamique de déstockage très progressive tout au long de la période de dessèchement. À granulométrie analogue, les petites différences de déstockage entre l'horizon A et $\mathrm{S}$ sont liées à la différence de teneur en eau à la capacité au champ. Cette teneur est plus élevée pour l'horizon A en raison de la présence de matière organique. Finalement, les conditions de stress hydrique vont être plus précoces et plus intenses pour les régosols. Une courte période évapotranspiratoire suffira à entraîner une pénurie d'eau. Dans le cas des sols limoneux, le végétal va pouvoir bénéficier d'une ambiance hydrique plus favorable à son développement en dépit d'une situation desséchante prolongée.

Les possibilités de remontées capillaires ont été récemment étudiées par A. Follet (2005). Ce travail montre de manière évidente l'absence de gradients hydriques décroissants de la nappe vers la partie supérieure des sols. Les résultats obtenus démontrent même le contraire (fig. 4b). Entre -60 et $-40 /-20 \mathrm{~cm}$, la teneur en eau décroît puis augmente entre ce dernier niveau et la sub-surface. Il n'apparaît pas de liens hydriques entre la nappe phréatique et les sables dunaires sus-jacents ni entre ces derniers et les sols limoneux. Si le contexte hydro-géologique local se prête à des remontées capillaires sous régime permanent (Hillel, 1974), cela n’est factuellement pas vérifié. 


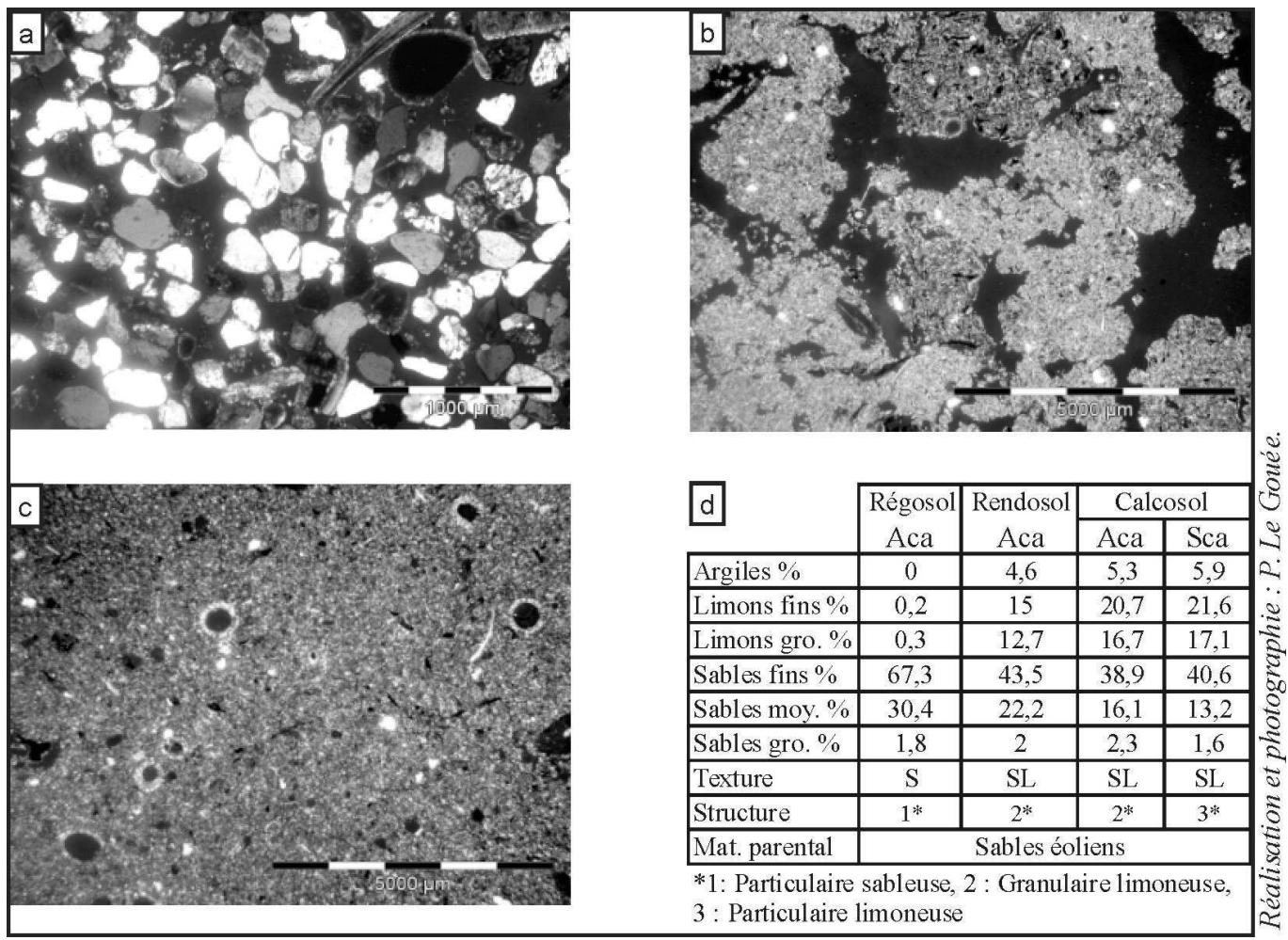

Figure 3 : Structures des sols et résultats granulométriques Structures of the soils ans particles-size results a. Structure particulaire grossière du régosol, b. Structure granulaire du rendosol et calcosol (Aca), c. Structure particulaire fine du calcosol (Sca), d. Données générales.

a. Coarse single grain structure of the regosol, $b$. Silty granular structure of rendosol and calcosol (Aca), $c$. Fine single grain structure of calcosol (Sca), d. Global data.

À l'exemple du calcosol, l'alcalinité des sols limoneux conduit à une saturation du complexe adsorbant en calcium (fig. 4c). La présence significative de calcaire total (supérieur à $30 \%$ ) et actif (oscillant autour de $10 \%$ ) est signalée tant pour l'humus que pour les horizons du solum. Cependant, nous ne pouvons pas parler de sol hyper-calcaire (Baize et Girard, 1995). Le rapport $\mathrm{C} / \mathrm{N}$ des sols limoneux traduit une minéralisation rapide de la matière organique (fig. 4c). Le stock de carbone organique, inférieur à $8 \%$, ne permet pas de qualifier les horizons A des sols limoneux d'hémi-organiques. L'accumulation de matière organique est également limitée dans la couche d'humus. Les teneurs en Fer libre et plus encore le rapport en Fer total/Fer libre du calcosol (fig. 4c) obtenus à partir d'échantillons prélevés au début du printemps 2005 attestent de concentrations très faibles du fer échangeable.

\section{Discussion}

\section{L'ORIGINE DES LIMONS}

La grande originalité des sols du massif dunaire d'Hatainville tient à la présence de couvertures pédologiques dotées d'abondantes particules limoneuses. À ce jour, les publications relatives aux sols des dunes côtières que nous avons consultées ne font pas état de cette particularité. Ces sols 


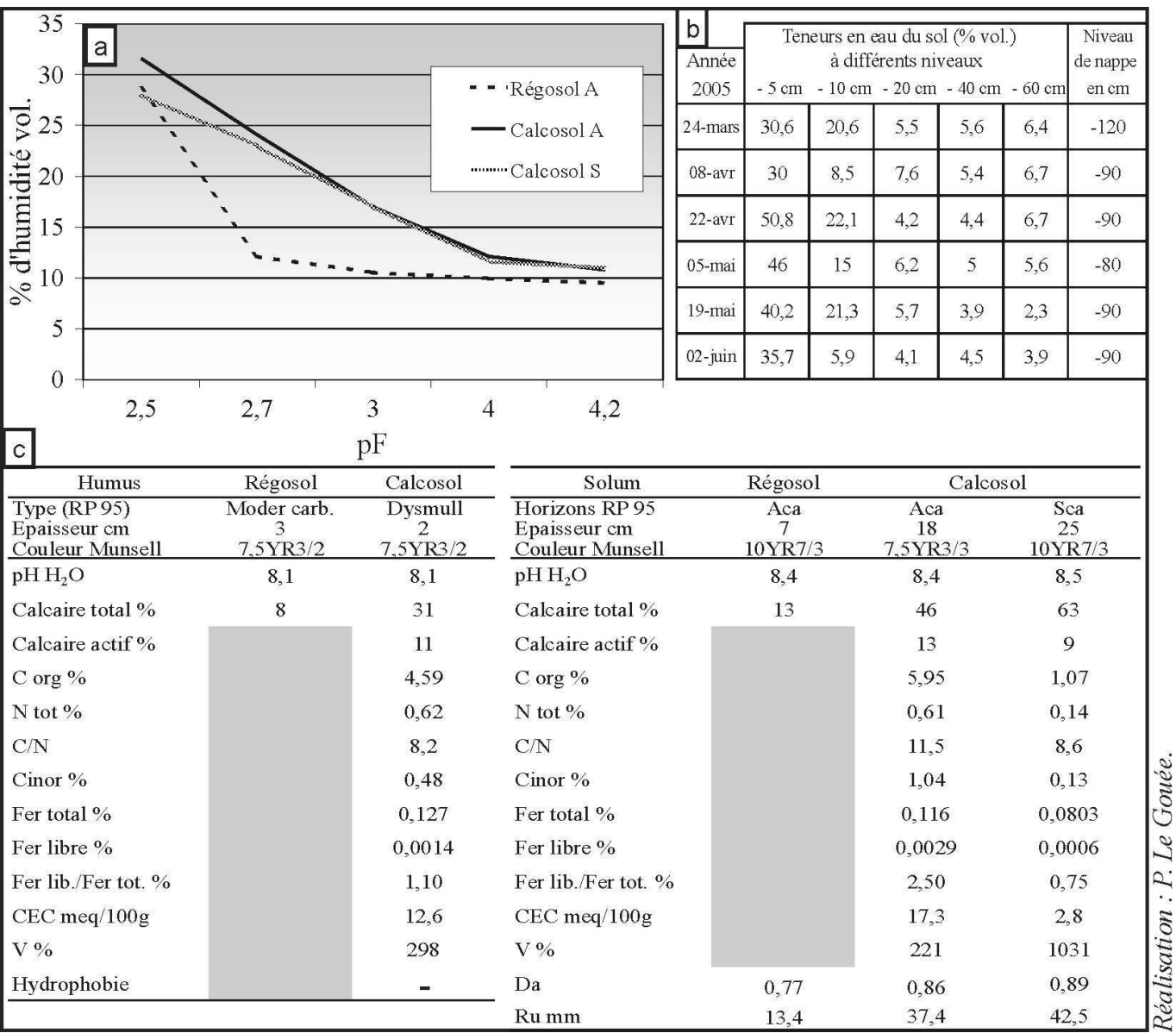

Figure 4 : Données hydriques et chimiques du régosol sableux et du calcosol limoneux Moisture and chemical data of sandy Regosol and silty Calcosol

a. Courbes d'humidités caractéristiques, b. Profils hydriques du calcosol, c. Données chimiques et réserves utiles. $a$. Characteristic moistures curves, $b$. Moisture profiles of Calcosol, c. Chemical data and useful available waters.

limoneux sont répartis sans logique apparente dans les dépressions situées dans les secteurs de dunes basses et de dunes paraboliques. La cartographie des dépressions sableuses et limoneuses (fig. 5) montre un positionnement des sols limoneux entre le secteur médian des dunes paraboliques et la falaise morte. Cependant, ces sols sont également présents au niveau des dunes basses, à proximité des dunes vives.

L'étude granulométrique des rendosols et calcosols révèle des distributions tri-modales (fig. 6a). Le premier mode est très étalé dans la fraction des limons $(2-63 \mu \mathrm{m})$, le deuxième s'apparente à la fraction 150-500 $\mu \mathrm{m}$ et le dernier, nettement moins significatif que les précédents, se situe entre 900 et $1400 \mu \mathrm{m}$. L'analyse microscopique des lames minces des sols limoneux permet de préciser la nature de ces trois modes. Si le mode intermédiaire traduit la présence d'éléments détritiques correspondant aux grains de quartz qui composent l'essentiel du sable dunaire du massif d'Hatainville, les premier et dernier modes renvoient respectivement à un matériel biogène et bio-détritique (fig. 6b). L'observation d'oospores de characées (fig. 6b et fig. 6d) témoigne de l'existence passée de 


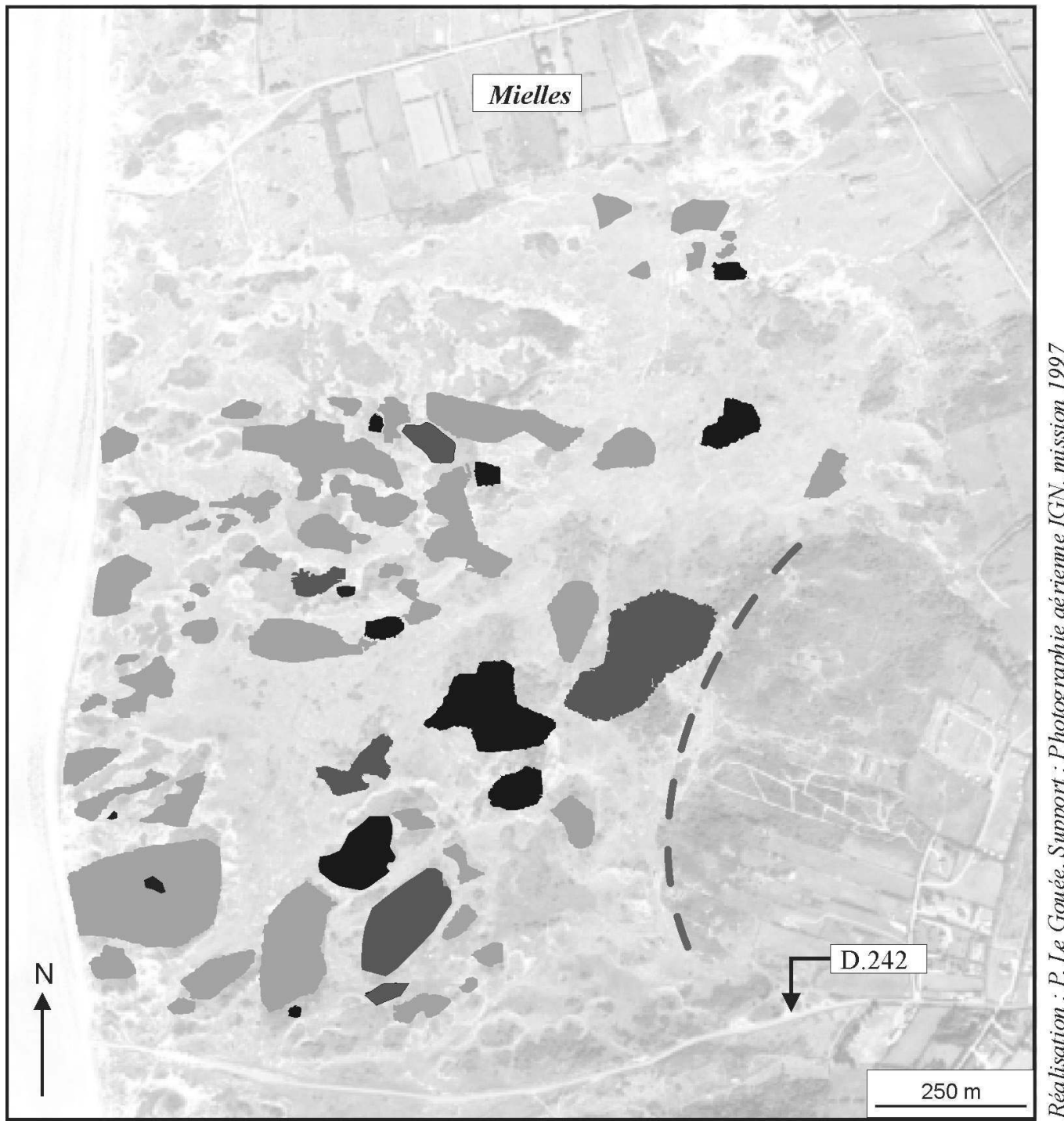

Figure 5 : Localisation des dépressions humides sableuses et limoneuses du massif d'Hatainville d'après A. Folley (2005).

Location of sandy and silty dune slacks in the Hatainville massif according to A. Folley (2005).

Pointillés : Talus (falaise morte), gris moyen : Dépressions à sols sableux, gris foncé : Dépressions à sols sableux et limoneux, noir : Dépressions à sols limoneux.

Dotted line: Slope (paleocliff), medium grey: Dune slacks with sandy soils, dark grey : Dune slacks with sandy and silty soils, black Dune slacks with silty soils.

charophytes (fig. 6c), plantes aquatiques calcaires, dont les organes reproducteurs (oospores) constituent les derniers témoignages identifiables. Lorsqu'ils sont bien conservés (fig. 6d), leur taille peut atteindre et dépasser les $1000 \mu \mathrm{m}$. Associés à la présence de quelques grains de sables grossiers, ces éléments expliquent l'apparition d'un troisième mode dans les distributions granulométriques. Lorsque les oospores se dégradent par fragmentation (fig. 6e), elles participent à la fabrication de particules de taille limoneuse. Cette fraction granulométrique, qui résulte également du démantè- 
lement des rameaux végétatifs des charophytes dont la structure originelle a complètement disparu d'après l'analyse microscopique, contribue à la formation d'une matrice limoneuse calcaire (fig. 6b). L'imbrication des sables dans la matrice limoneuse souligne la concomitance du processus de dépôts des fragments charophytiques et du processus de piégeage des sables fins éolisés dans les dépressions humides. La variation du rapport sable/limons qui se manifeste dans les résultats granulométriques est la traduction de la fréquence et la durée de l'occupation des creux dunaires par les characées ainsi que l'expression de la fréquence et la durée de la fourniture en sables par le vent. Cette remarque peut également être formulée à propos de l'épaisseur des sols sablo-limoneux.

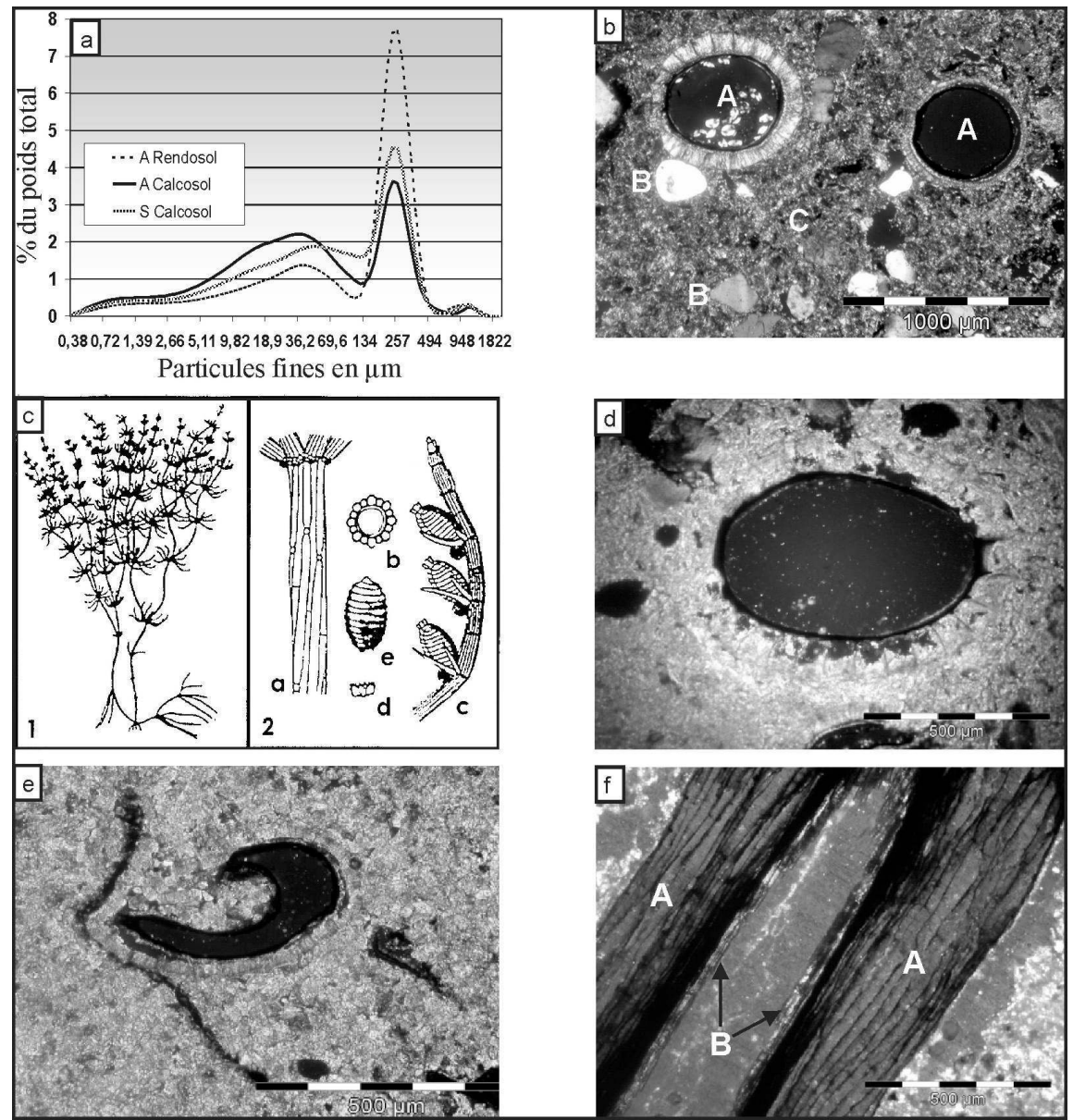

Figure 6 : Origine des limons et caractéristique pédologique des sols limoneux

Origin of silts and pedofeature of silty soils

a. Distribution des particules minérales élémentaires, b. Lame mince de Aca du Calcosol( Oospores de characées en coupe polaire A, grains de quartz B et matrice limoneuse calcitique C), c. Structure des characées d'après M. Feist-Castel, 1984 (1. rameau végétatif incrusté de carbonate de calcium, 2a. section de rameau, $2 \mathrm{~b}$. coupe polaire d'un rameau, 2c. rameau avec organe reproducteur, $2 \mathrm{~d}$. coronule, 2e. Oospore), d. Oospore en coupe équatoriale, e. Oospore dégradée en coupe équatoriale, f. Pseudomorphose partielle d'un tissu végétal A par la calcite B. Photographies LPA.

a. Distribution of simple mineral particles.b. Thin section of Calcosol's Aca (Oospores of characeae by polar section A, quartz grains Band calcitic silty matrix C), c. Characeaea structuration according to M. Feist-Castel, 1984 (1. Vegetative branch encrusted with calcium carbonate, $2 a$. Section of branch, $2 b$. Polar cut of a branch, 2c. Branch with reproductive body, $2 d$. Coromule, 2e. Oospore), d. Oospore out of equatorial cut, e. Oospore degraded out of equatorial cut, f. Partial pseudomorphosis of plant tissue (A) by calcite (B). LPA photographs. 
Patrick Le Gouée, Yves Petit-Berghem, Audrey Folley

\section{L'INTÉRÊT ÉCOLOGIQUE DES SOLS LIMONEUX}

Les dépressions limoneuses sont caractérisées par la présence d'espèces indicatrices des pelouses pionnières des dépressions et prairies humides dunaires. Les pelouses pionnières correspondent à des végétations de gazons herbacés ras à très ras, inondés pendant l'année. On y retrouve le Mouron délicat, Anagallis tenella, le Léontodon faux-pissenlit, Leontodon taraxacoides, la Petite Centaurée, Centaurium erythraea, la Sagine noueuse, Sagina nodosa, protégée au niveau régional, la Littorelle des Lacs, Littorella uniflora, protégée au niveau national et l'Ache rampante, Apium repens, visée dans l'annexe II de la directive "Habitats» (fig. 7a et fig. 7b). Ces pelouses sont colonisées par des végétations vivaces plus hautes et plus denses correspondant aux végétations prairiales inondables. L’Agrostide stolonifère, Agrostis stolonifera, la Menthe aquatique, Mentha aquatica, la Potentille rampante, Potentilla reptans, ou bien encore des espèces rares comme le Jonc du Tonnelier, Juncus effusus (fig. 7c), l'Euphraise quadrangulaire, Euphrasia tetraquetra, l'Odontite rouge, Odontites Vernus, et la Germandrée des marais, Teucrium scordium, composent ces prairies dunaires. Dans leur très large globalité, ces espèces sont qualifiées de neutrophiles à calciclines et d'hygrophyles à amphibie.

Les dépressions dunaires sont associées à l'idée d'humidité et contrastent en cela avec les importants déficits en eau observés sur les autres secteurs topographiques du massif sableux. Cependant, l'abaissement des eaux souterraines au début du printemps expose les sols à des conditions évapotranspiratoires favorisant leur dessèchement rapide. La connaissance de la réserve utile est donc très utile pour définir la valeur de l'offre en eau du sol exploitée par le couvert végétal. À ce titre, les résultats soulignent des écarts considérables entre un régosol sableux et un calcosol limoneux (fig. 4c). Les différences de comportements hydriques entre régosols et sols limoneux peuvent-elles être un élément discriminant dans la composition et l'évolution de la végétation des dépressions humides en présence d'une nappe phréatique locale? La relation entre la nappe d'eau souterraine et la réserve hydrique du sol dépend, d'une part, de l'existence de remontées capillaires et, d'autre part, de la position de la nappe phréatique par rapport au sol. Or, le suivi des profils hydriques témoigne de l'absence de remontées capillaires sous régime permanent. Par conséquent, lorsque la nappe d'eau n'est plus en connexion avec les sols dunaires, les valeurs et dynamiques hydriques de RU peuvent constituer des facteurs discriminants dans la composition et l'évolution de la végétation des dépressions humides. De ce fait, la présence d’une fraction limoneuse dans les sols revêt un intérêt écologique tout particulier au regard des espèces hygrophiles et aquatiques les plus précieuses du massif dunaire d'Hatainville (Ache rampante, Apium repens, Littorelle des lacs, Littorella uniflora), espèces dont la présence est étroitement associée aux sols limoneux. Si, en période de décharge de la nappe d'eau, l'alimentation de la réserve utile des sols du massif dunaire ne peut pas s'obtenir par les remontées capillaires, les tests de mouillabilité à l'eau sur les humus montrent qu'elle peut être assurée par une infiltration assez rapide des précipitations (fig. 4c). Les temps d'infiltration, de 3 secondes à 1 minute, témoignent d'une hydrophobie faible à modérée.

Si l'eau dans le sol et son absence agissent sur l'activité végétative, il en va de même concernant la réserve trophique des couvertures pédologiques. Celle-ci est définie par la nature et la charge en éléments nutritifs assimilables par les végétaux. À ce propos, la mesure du pH révèle une saturation du complexe adsorbant en calcium. Cela n'est pas surprenant compte tenu de la nature des limons. L'analyse microscopique de lames minces témoigne de la présence de pseudomorphoses de tissus végétaux par la calcite (fig. 6f). Les sols limoneux sont donc soumis au processus de carbonatation sous climat lessivant. Cette pédogenèse est associée à des sols juvéniles en milieu alcalin (Wilson, 1960 ; Lammerts et al., 2001). Ces pseudomorphoses expriment le développement d'une carbonatation secondaire, traduisant l'existence de conditions de sécheresse édaphique prolongées. En l'état actuel des conditions hydrologiques, le processus de décarbonatation dans les rendosols et calcosols ne peut s'opérer, ce qui exclut toute tendance à l'acidification. D’après les travaux de G. J. Kenoyer et M. P. Anderson (1989), cette alcalinité peut-être entretenue lors des phases de remontée de la nappe. Ainsi, en période de submersion, le fonctionnement hydrologique s'apparente à celui des 
lacs, avec une recharge d'un côté et une infiltration de l'autre. Lorsque la nappe se trouve dans des sables dunaires calcaires, ce qui est le cas sur le site d'Hatainville, les eaux souterraines se chargent de carbonates de calcium. Les dépressions capturent alors les ions calcium transportés par la nappe (Grootjans et al., 1998). La présence de calcaire actif dans les sols limoneux conduit à apporter aux végétaux un stock abondant de ce minéral qui leur est indispensable. Toutefois, l'absence d'une trop forte alcalinité autorise la présence d'espèces végétales non calcicoles dans les dépressions humides, ce qui contribue ainsi à favoriser la biodiversité végétale.

Si la nappe d'eau intervient dans le niveau d'alcalinité des sols limoneux, elle joue également un rôle important dans la minéralisation de la matière organique. Sous conditions alcalines, il apparaît que les flux d'eau générés par la remontée de nappe stimulent une minéralisation rapide de la matière organique (Van Beckhoven, 1995). En conséquence, la décomposition rapide de la matière organique favorise le recyclage des éléments nutritifs dans l'écosystème et soutient efficacement le développement des sols limoneux (Grootjans et al., 1998). Cette décomposition est également soutenue en période de sécheresse estivale par la mise en contact de la végétation résiduelle avec l'oxygène et les radiations solaires.

Le caractère inondable des dépressions humides limoneuses entraîne une saturation en eau des sols pendant une période plus ou moins longue. Ces conditions anoxiques vont entraîner une augmentation des teneurs en fer et manganèse échangeables (Jones, 1973). Selon cet auteur, la concentration du fer et du manganèse sous forme échangeable est l'un des facteurs déterminants du développement de la végétation dans les dépressions dunaires partiellement inondées car cette concentration peut engendrer des niveaux toxiques. Ces résultats peuvent s'expliquer, tout d'abord, par l'absence de conditions anoxiques pendant l'hiver dans les sols limoneux du massif dunaire. Ensuite, le contexte alcalin de ces sols est incompatible avec des valeurs élevées de Fer échangeable. Enfin, les faibles taux de carbone organique obtenus dans les sols limoneux ne favorisent pas de fortes concentrations en Fer échangeable puisqu'il a été démontré que ces dernières-ci sont corrélées avec des teneurs importantes en matière organique des sols (Jones, 1973). Les teneurs en Fer échangeables dans les sols limoneux ne semblent donc pas intervenir dans la composition de la végétation.

Finalement, l'intérêt écologique des sols limoneux relève, d'une part, de leurs propriétés hydriques qui, en limitant les conditions de stress hydrique trop sévères lors du retrait de la nappe, permet la présence et le maintien des espèces hygrophiles les plus intéressantes. Cet intérêt réside, d'autre part, dans l'absence de fortes contraintes trophiques qui assure le développement de nombreuses espèces végétales plus communes. Par conséquent, la richesse biologique des sols limoneux se justifie à la fois par la présence spécifique d'espèces végétales rares et protégées et par l'existence d'une forte diversité floristique.

\section{LES PROBLÈMES POSÉS ET LA GESTION PRÉCONISÉE CONCERNANT LES SOLS LIMONEUX}

L'étude microscopique a montré que les charophytes sont à l'origine des limons. Il s'agit de plantes aquatiques identifiées aux habitats correspondant aux herbiers aquatiques des mares, flaques et plans d'eau stagnante arrière-dunaires, au moins temporairement en contact avec la nappe phréatique (Natura 2000, 1999). La cartographie des dépressions humides du massif d'Hatainville (fig. 5) souligne le caractère peu banal des dépressions dunaires limoneuses puisqu'elles représentent moins de $10 \%$ du total. On retrouve un peu plus fréquemment des dépressions possédant à la fois des sols limoneux et des régosols sableux. Dans ce cas de figure, les sols limoneux sont toujours positionnés dans les secteurs les plus bas des dépressions. Quoi qu'il en soit, les rendosols et calcosols limoneux se situent préférentiellement dans la partie médiane du massif, depuis le complexe de dunes paraboliques et longitudinales jusqu'aux dunes basses dominées par le talus. Cette localisation doit être mise en relation avec le type d'habitat spécifique aux characées. D’après les Cahiers habitats (Natura 2000, 1999), ces algues calcaires sont inféodées aux mares dunaires. Celles-ci sont caractérisées par des eaux stagnantes arrière-dunaires, au moins temporairement en 
contact avec la nappe phréatique, peu profondes, pouvant s'échauffer rapidement et reposant sur des fonds de nature sablonneuse. Ces mares sont soumises parfois à des fluctuations importantes des niveaux d'eau entraînant presque leur assèchement. Il est important de relever cette dernière caractéristique hydrologique qui souligne la nécessité d'un remplissage pérenne des dépressions dunaires. Par conséquent, compte tenu du contexte hydrologique local, l'alimentation en eau des dépressions à characées est associée à la surface libre de la nappe phréatique dont la hauteur permet de maintenir un état de submersion des creux dunaires. Autrement dit, les sols limoneux témoigneraient du positionnement dans le massif dunaire des situations durables de submersion par la nappe d'eau. L'absence de données piézométriques sur plusieurs années ne nous permet malheureusement pas de valider cette hypothèse. Néanmoins, le lien établi entre les characées et la présence d'eau stagnante soulève une question fondamentale : les characées qui composent la fraction limoneuse des rendosols et calcosols sont-elles héritées ou actuelles? Au sens strict du terme selon les Cahiers habitats, il n'existe qu'une seule mare dunaire dans le massif d'Hatainville. Par ailleurs, les mares temporaires sont rares et ne correspondent pas systématiquement aux dépressions limoneuses. En conséquence, les conditions hydrologiques actuelles ne sont pas favorables à la présence des characées. Par ailleurs, l'analyse microscopique des lames minces souligne une diagenèse partielle du matériel biogène (fig. 6d et fig. 6e) et une pédogenèse avancée dans les dépôts limoneux (fig. 6f). Ces processus témoignent de la nécessité de conditions régulièrement aérobiques depuis de nombreuses années. Ainsi, nous pensons que les dépressions limoneuses sont l'expression de mares dunaires anciennes qui ont évolué vers des types d'habitat moins aquatiques comme les prairies humides dunaires, les bas-marais dunaires et les pelouses pionnières. Ces sols seraient alors un indicateur d'un changement du régime hydrologique local depuis de nombreuses années, marqué par un abaissement durable du niveau de la surface libre de la nappe phréatique. On doit alors s'interroger sur les raisons de cet abaissement. L'origine est-elle naturelle en lien avec l'évolution du climat? Les sources d'alimentation de la nappe phréatique ont-elles été en partie captées ou détournées par l'homme? Une étude sera menée prochainement sur ce sujet.

L’intérêt écologique des dépressions dunaires limoneuses relève prioritairement de la présence de cortèges végétaux dans lesquels apparaissent des espèces assez rares à rarissimes. Ces cortèges correspondent à des espèces qui interviennent dans une dynamique de conquête ou de reconquête du milieu. Elles représentent donc l'étape initiale de la dynamique d'évolution de la végétation sur le massif dunaire. De ce fait, ces espèces sont amenées à disparaître au profit d'autres unités végétales, entraînant une diminution de la biodiversité et l'établissement de formations ligneuses de type saulaie haute de Saules roux cendrés (De Raeve, 1989). Cette tendance observée sur le site d'Hatainville ne fait que confirmer l'évolution très importante à la régression des pelouses pionnières et des prairies humides dunaires en France (Natura 2000, 1999). Sur le massif dunaire d'Hatainville, les facteurs de régression relèvent principalement de la fermeture des dépressions par les formations buissonnantes et arbustives et de l'abaissement constaté de la surface libre de la nappe phréatique. Ce dernier facteur est plus préoccupant que la colonisation végétale car les moyens d'intervention sont nuls et les conséquences sur les habitats sont très dommageables. C'est ainsi qu'aux Pays-Bas, les communautés de plantes pionnières basiphiles sont menacées de disparition suite à un retrait des nappes d'eau (Lammerts et al., 2001). Dans la mesure où le temps de reproduction des pelouses pionnières pérennes oscille entre 3 et 8 ans, période couvrant la germination jusqu'à la première floraison (Ernst et van der Ham, 1988), toute modification du régime hydrologique traduisant un abaissement de la nappe au cours de cette phase végétative compromettra le processus de reproduction. La réserve utile et son comportement dans les sols limoneux du massif d'Hatainville peut pallier la décharge de la nappe et satisfaire les besoins des espèces méso-hygrophiles et hygrophiles. Cependant, cela ne sera pas suffisant pour les espèces aquatiques à amphibie menacées alors de disparition. Par ailleurs, l'abaissement de la nappe d'eau renforce le dessèchement des sols, ce qui tend à modifier les processus pédogénétiques (Sival et Grootjans, 1996). Si les conditions climatiques demeurent peu lessivantes, la carbonatation (primaire et secondaire) prévaudra. Cela renforcera l'alcalinité des sols, engendrant ainsi un blocage progressif de la minéralisation de la matière organique et une 


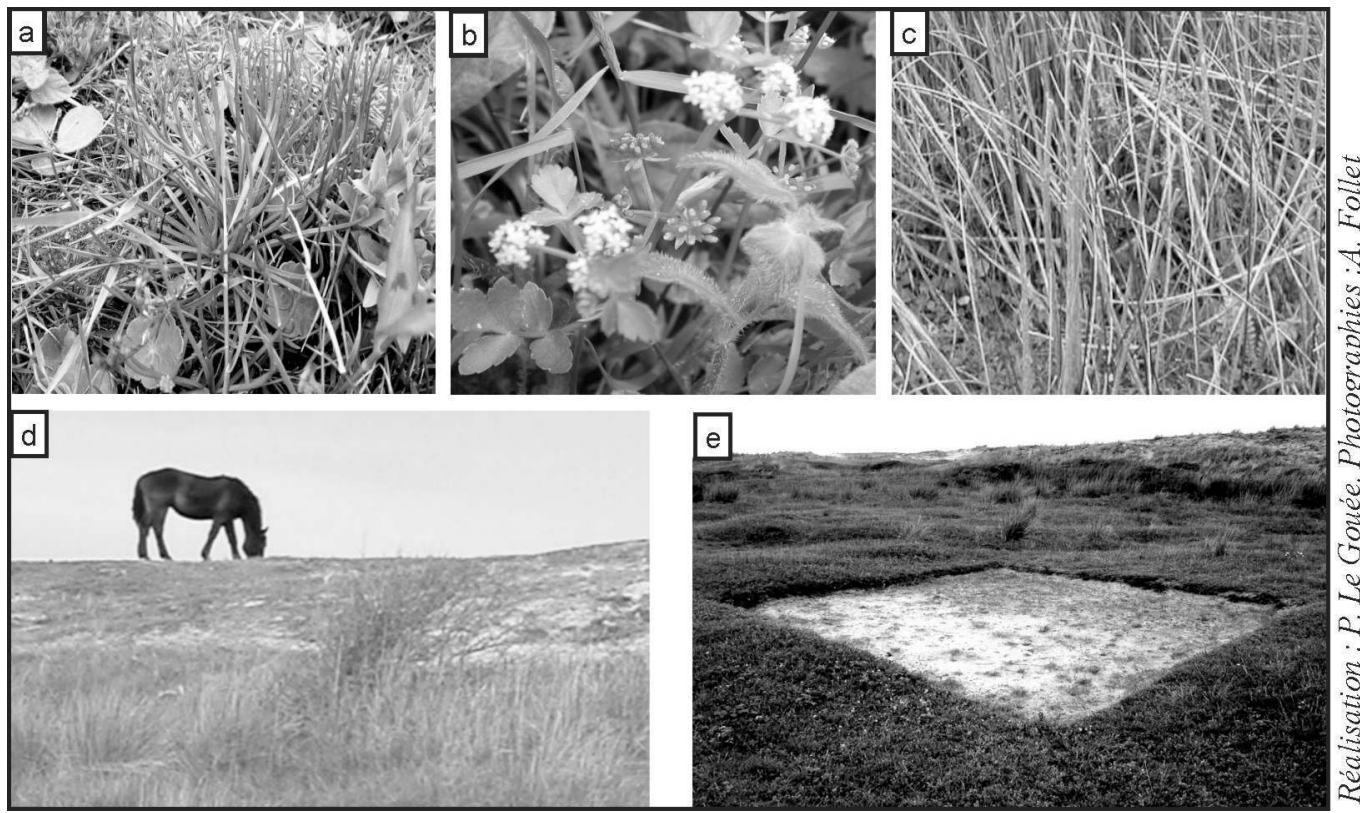

Figure 7 : Espèces hygrophiles rares et modes de gestion des dépressions dunaires Scarce hygrophile species and types of dune slacks management

a. Littorelle des lacs, Littorella uniflora, b. Ache rampante, Apium repens, c. Jonc du tonnelier, Scirpus lacstris, d. Pâturage extensif, e. Étrépage.

a. Littorella uniflora, b. Apium repens, c. Scirpus lacstris, d. Extensive grazing, e. Shallow cleaning.

réduction de l'offre trophique des sols. Ce blocage sera également soutenu par l'absence des flux d'eau qui accompagnent la remontée de la nappe (Kenoyer et Anderson, 1989). En revanche, en présence d'un contexte lessivant, le processus de décarbonatation déclenchera une acidification des sols (Sival, 1996). Par conséquent, quels que soient les scénarii climatiques envisagés, l'évolution pédogénétique provoquée par une modification durable du régime hydrologique de la nappe deviendrait un facteur décisif dans le développement des espèces des dépressions dunaires (Grootjans et al., 1997; Berendse et al., 1998).

La colonisation par les vivaces hautes et l'embroussaillement constituent la première menace à laquelle sont confrontées les plantes pionnières et prairiales des dépressions humides limoneuses. La lutte contre la prolifération d'espèces envahissantes dans le massif d'Hatainville se traduit par la mise en place du pâturage extensif au moyen d'un cheptel équin (fig. $7 \mathrm{~d}$ ). Confrontés au même type de problème, les Néerlandais ont montré que cette forme de pâturage ne suffit pas à stopper la prolifération des vivaces hautes mais permet néanmoins le maintien des espèces les plus intéressantes (Kooijman et Van der Meulen, 1996). À ce titre, cette démarche conservatoire peut être considérée comme un outil de gestion satisfaisant. Dernièrement, cette action s'est accompagnée d'une opération destinée à introduire une population de léporidés. Les conséquences positives d'une telle mesure sont encore discutées dans la communauté scientifique. Il est reconnu que le pâturage des prairies humides par les lapins et lièvres enraye la prolifération de plantes envahissantes. En outre, ces léporidés, en remuant le sol, favorisent l'intégration de l'azote dans le sol et participent ainsi à l'amélioration de la disponibilité trophique des horizons pédologiques (Grootjans et al., 1998). Ce travail du sol va également remobiliser les stocks de graines enfouis et favoriser le renouvellement des espèces (Gàbor et al., 2005). Cependant, le pâturage de ces animaux réduit la biomasse de surface. Il modifie ainsi la contribution de la litière dans le sol, 
ce qui s'exprime par une moindre accumulation de matière organique. Or celle-ci est essentielle pour maintenir la pédogenèse et garantir des conditions trophiques satisfaisantes aux végétaux (Grootjans et al., 1998).

La lutte contre l'évolution de la végétation qui conduit à une fermeture des dépressions réside dans la pratique du débroussaillement au centre des dépressions tout en conservant les bosquets périphériques servant d'abri pour la faune. Le débroussaillage des fourrés revêt l'intérêt d'écarter des espèces communes comme le Troène, Ligustrum vulgare, la Ronce, Rubus, le Saule rampant, Salix repens, et le Saule roux cendré, Salix atrocinerea, au profit d'espèces herbacées à plus forte valeur patrimoniale. En outre, les formations arbustives assèchent rapidement les dépressions (van Dijk et Grootjans, 1993). Cette opération permet de maintenir plus longtemps de bonnes conditions hydriques pour l'alimentation d'espèces prairiales ou pionnières hygrophiles et mésohygrophiles. Quelques années après, une fauche d'entretien et/ou un étrépage partiel (fig. 7e) est entrepris. La fauche est une pratique reconnue pour conserver les espèces pionnières et prairiales menacées. Toutefois, cette opération n'est pas sans conséquence sur certaines plantes à forte valeur patrimoniale comme les orchidées (Grootjans et al., 1998). L'étrépage, qui se traduit par un décapage des premiers centimètres de sol, modifie radicalement le contexte édaphique. Cette démarche entraîne la disparition de la litière qui alimente le sol en matière organique et tronque substantiellement l'horizon A. Dans le cas de sols peu épais, l'étrépage peut conduire à une complète disparition du matériel édaphique. Compte tenu de la forte valeur écologique des sols limoneux, cette intervention humaine doit être mûrement réfléchie. Si, comme nous le croyons, les particules biogènes s'apparentent à des dépôts hérités, l'étrépage engendrera une perte définitive d'une partie des couvertures pédologiques limoneuses. Dans la mesure où l'Ache rampante, Apium repens, et la Littorelle des lacs, Littorella uniflora, semblent inféodées aux dépressions limoneuses, l'étrépage pourrait modifier de manière irréversible leur patrimoine écologique.

La modification du régime hydrologique représente la seconde menace. Les conditions de développement des espèces pionnières et prairiales sont étroitement liées à la présence des nappes et à la fluctuation des niveaux d'eau relevant des conditions climatiques locales (Lammerts et al., 2001). Pour pallier l'abaissement du niveau de la nappe phréatique et permettre l'existence de mares permanentes et temporaires nécessaires aux espèces patrimoniales mais aussi à l'alimentation en eau du cheptel équin, le rajeunissement et le recreusement des dépressions dunaires a été envisagé dans des dépressions sableuses, conformément aux propositions de gestion nationales (Natura 2000, 1999). Au regard de ce que nous avons dit à propos de l'origine et de l'intérêt écologique des sols limoneux, ce type d'opération doit être exclu. En revanche, il serait intéressant d'entreprendre une expérimentation de cette nature dans des dépressions sableuses afin d'étudier les possibilités d'implantation des characées. Dans cette perspective et dans la mesure où les algues calcaires sont très sensibles à l'eutrophisation, il serait nécessaire de définir un périmètre de protection interdisant la présence de bétail.

\section{Conclusion}

Le long des côtes européennes, les systèmes dunaires sont caractérisés par des ambiances hydriques et trophiques variées en lien avec les spécificités géomorphologiques et hydrologiques locales. Cela favorise la création d'habitats à grande valeur patrimoniale qui sont généralement associés à la présence de dépressions humides. Partout en Europe, ces habitats régressent à cause des processus de colonisation, d'embroussaillement et d'abaissement des niveaux de nappe. Cette tendance est également perceptible dans le massif dunaire d'Hatainville où les pelouses pionnières et les prairies humides d'intérêt écologique majeur sont fortement menacées par les ligneux. De nombreuses opérations conservatoires sont menées par les organismes en charge de la gestion du massif. À la demande de ces gestionnaires, une étude pédologique a été réalisée dans les dépressions humides afin de préciser leurs liens avec les espèces pionnières et prairiales les plus intéressantes. Cette étude a permis de mettre en évidence l'existence de sols limoneux 
portant exclusivement les espèces hygrophiles et aquatiques les plus précieuses comme l'Ache rampante ou la Littorelle des lacs. L'étude analytique de ces sols montre que le matériel limoneux correspond à la diagenèse des characées dont le caractère hérité est souligné par la pédogenèse qui s'est développée à ses dépens. La présence de ces algues calcaires contrôle largement les propriétés physiques, hydriques et chimiques des sols limoneux. Les conditions hydriques et trophiques apparaissent remarquables dans ce site dunaire et soutiennent la présence des espèces hygrophiles rares. Le caractère hérité de la fraction limoneuse semble attester, comme partout en Europe de l'Ouest et du Nord, une tendance durable à l'abaissement de la nappe phréatique. Dans ce contexte hydrologique, le processus de carbonatation qui affecte les sols limoneux risque d'évoluer en lien étroit avec les conditions climatiques. Un renforcement de la carbonatation ou une acidification sont envisagées. Les transformations géochimiques avancées entraineraient inévitablement une modification dans la composition des espèces végétales basses. Si la lutte pour la préservation des plantes pionnières et prairiales passe par des actions de décolonisation et de débroussaillement, les moyens pour assurer la présence temporaire et permanente d'eau dans les dépressions passent par leur recreusement. Une telle démarche doit cependant être exclue dans les dépressions limoneuses compte-tenu de leur spécificité.

\section{Bibliographie}

Baize (D.), Girard (M.-C.), 1995. - Référentiel pédologique, 1995, Paris, INRA, 332 p.

Berendse (F.), Lammerts (E. J.), OlfF (H.), 1998. - « Soil organic matter accumulation and its implications for nitrogen mineralization and plant species composition during succession in coastal dune slacks », Plant Ecol., 137, p. 71-78.

De Raeve (F.), 1989. - «Sand dune vegetation and management dynamics », Perspectives in Coastal Dune Management, Éditions SPB Academic Publishing, p. 99-109.

Dekker (L. W.), Jungerius (P. D.), 1990. - «Water repellency in the dunes with special reference to the Netherlands », Catena (suppl.) 18, p. 173-183.

Ducerf (G.), Thiry (C.), 2003. - Les plantes bio-indicatrices. Guide de diagnostic des sols, éditions Promonature, $278 \mathrm{p}$.

ERnst (W. H. O.), VAn der Ham (N. F.), 1988. - « Population structure and rejuvenation potential of Schoenus nigricans in coastal wet dune slacks », Acta Bor Neerl., 37, p. 451-465.

Favennec (J.), 1998. - Guide de la flore des dunes littorales, Bordeaux, Sud-Ouest/Office National des Forêts, $167 \mathrm{p}$.

Feist-Castel (M.), 1984. - Les charophytes dans le Paléogène du sud de la France (Provence, Languedoc, Aquitaine), Thèse, Université des Sciences et Techniques du Languedoc, 82 p.

FolLey (A.), 2004. - Contribution à l'étude de massif dunaire d'Hatainville : le rapport sol/végétation dans les dépressions humides intra dunales, Mémoire de maîtrise, université de Caen Basse-Normandie, 173 p.

—, 2005. - Les sols limoneux des dépressions humides du massif dunaire d'Hatainville : une ressource naturelle fragile à forte valeur écologique, Mémoire de Master recherche, université de Caen Basse-Normandie, $179 \mathrm{p}$.

Foucault (B. de), 1984. - Systémique, structuralisme et synsystématique des prairies hygrophiles des plaines atlantiques françaises, Thèse d'État, Université de Rouen et de Lille 2, 2 tomes, 675 p.

GÀBor (M.), PApP (M.), Tothmeresz (B.), 2005. - « Impact of management on vegetation dynamics ans seed bank formation of inland dune grassland in Hungary », Flora, p. 296-306.

GéHu (J.-M.) et GéHu-Franck (J.), 1982. - La végétation du littoral Nord-Pas-de-Calais (essai de synthèse), Région Nord-Pas-de-Calais/CREPIS, vol. 1, 361 p.

Grootjans (A. P.), Ernst (W. H. O.), Stuytsand (P. J.), 1998. - « European dune slacks : strong interactions of biology, pedogenesis and hydrology », TREE, vol. 13, n³, p. 96-100. 
Patrick Le Gouée, Yves Petit-Berghem, Audrey Folley

Grootjans (A. P.), Van den Ende (F. P.), Walsweer (A. F.), 1997. - "The role of microbial mats during primary succession in calcareous dune slacks : an expérimental approach”, J. Coast. Conserv., 3, p. 95-102.

Guillore (P.), 1980. - Fabrication mécanique et en série de lames minces de sols, Institut national d'Agronomie, Paris-Grignon (polycopié).

Hillel (D.), 1974. - L'eau et le sol, principes et processus physiques, Louvain, Ed. Vander, 288 p.

Jabiol (B.), Brêthes (A.), Ponge (J.-F.), Toutain (F.), Brun (J.-J.), 1995. - L'Humus sous toutes ses formes, École Nationale du Génie Rural, des Eaux et des Forêts, Nancy, France, 63 p.

Jones (R.), 1973. - « Comparative Studies of Plant Growth and Distribution in Relation to Waterlogging: VII. The Influence of Water-Table Fluctuations on Iron and Manganese Availability in Dune Slack Soils », The Journal of Ecology, vol. 61, nº 1, p. 107-116.

Kenoyer (G. J.), Anderson (M. P.), 1989 - « Groundwater's dynamic role in regulating acidity and chemistry in a precipitation lake», J. Hydrol., 109, p. 287-306.

Kooijman (A. M.), VAn Der Meulen (F.), 1996. - « Grazing as a control against "grassencroachment" in dry dune grasslands in the Netherlands », Landscape and Urban Planning, vol 34, Issues 3-4, p. 323-333.

Lammerts (E. J.), MaAs (C.), Grootjans (A. P.), 2001. - « Groundwater variables and vegetation in dune slacks », Ecological Engineering, vol. 17, Issue 1, p. 33-47.

LeVoy (F.), 1996. - Suivi de l'évolution des sites dunaires sur les côtes bas-normandes : année 1995, Laboratoire de Géologie Marine, Université de Caen et Conservatoire de l'Espace Littoral et des Rivages Lacustres, $90 \mathrm{p}$.

Livory (A.), Stallegger (P.), 2001. - Expertise faune et flore du massif dunaire d'Hatainville et du cap de Carteret, Conservatoire du Littoral et des Rivages Lacustres, 127 p.

Llamas (M. R.), 1990. - «Geohydrology of the eolian sands of the Doñana National Park (Spain)», Catena (suppl.), 18, p. 145-154.

NATURA 2000, 1999. - Manuel d'interprétation des habitats de l'Union Européenne, Commission Européenne, DG Environnement, 132 p.

Provost (M.), 1975. - Massif dunaire de Beaubigny. Site et végétation, Conservatoire de l'Espace Littoral et des Rivages Lacustres, 81 p.

—, 1998. - Flore vasculaire de Basse-Normandie, Presses Universitaires de Caen, 2 tomes, 902 p.

Sival (F. P.), Grootjans (A. P.), 1996. - « Dynamics of seasonal bicarbonate supply in a dune slack : effect on organic matter, nitrogen pool and vegetation succession », Vegetatio, 126, p. 39-60.

Sival (F. P.), 1996. - « Mesotrophic basiphilous communities affected by changes in soil properties in two dune slack chronosequences », Acta Bot. Neerl., 45, p. 119-131.

Van Beckhoven (K.), 1995. - Rewetting of Coastal Dune Stocks: Effects on Plant Growth and Soil Processes, Doctorate Thesis, Free University of Amsterdam, 135 p.

Van Dijk (H. W. J.), Grootjans (A. P.), 1993 - «Wet dune slacks: decline and opportunities », Hydrobiol., 265, p. 281-304.

VAN OORT (F.), 1984. - Géométrie de l'espace poral, comportement hydrique et pédogenèse. Application à des sols sous prairie et alpage, issus de l'altération calschiste sédimentaire en moyenne et haute montagne, Alpes du Nord (Baufortain), Thèse INA, Paris-Grignon, 273 p.

Wilson (K.), 1960. - « The Time Factor in the Development of Dune Soils at South Haven Peninsula, Dorset », The Journal of Ecology, vol. 48, n², p. 341-359.

Cet article a été reçu le 16 décembre 2005 et définitivement accepté le 27 mars 2006.

\section{REMERCIEMENTS}

Nous tenons à exprimer toute notre gratitude au SyMEL (Syndicat Mixte des Espaces Littoraux de la Manche) qui nous a autorisé à mener l'étude de pédologie dunaire sur le massif d'Hatainville et qui nous a apporté de précieuses informations concernant la biodiversité végétale et les pratiques de gestion du site. 